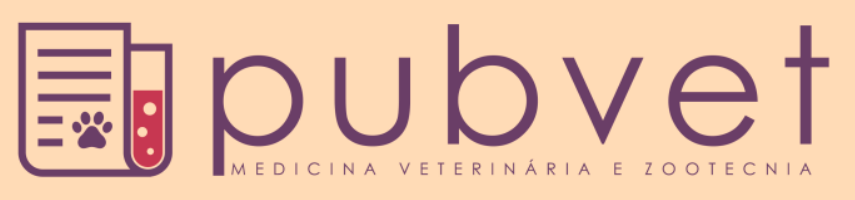

https://doi.org/10.31533/pubvet.v12n9a169.1-9

\title{
Ocorrência de parasitas gastrintestinais em fezes de cães coletadas em vias públicas do município de Valença - RJ
}

\author{
Beatriz Abdalla Ferraz de Barros $^{1} \bullet$, Josielle de Almeida Pereira ${ }^{1}$, Leanndro de Araújo \\ Barreto $^{1}{ }^{\ominus}$, Thayassan Costa dos $\operatorname{Santos}^{1}{ }^{\bullet}$, Lilian Cristina de Sousa Oliveira Batista \\ Cirne $^{2^{*} \bullet}$
}

${ }^{I}$ Discente no curso de Medicina Veterinária-Centro de Ensino Superior de Valença-RJ, Brasil.

${ }^{2}$ Professora adjunta no Centro de Ensino Superior de Valença-RJ, Brasil.

*Autor para correspondência: vet.lilianbatista@gmail.com

\begin{abstract}
RESUMO. Considerando a importância da infecção por parasitos gastrintestinais de cães, tanto no aspecto da clínica veterinária como no da saúde pública, estudos sobre a ocorrência desses endoparasitos, presentes em fezes de cães em vias públicas são necessários para se estabelecerem medidas de controle e profilaxia. Dessa forma, o presente estudo teve como objetivo avaliar a ocorrência de parasitas gastrintestinais presentes em fezes de cães coletadas em vias públicas do município de Valença -RJ no período de agosto a outubro de 2017. Um total de 63 amostras de fezes frescas de cães foram coletadas e encaminhadas ao Laboratório de Parasitologia e Doenças Parasitárias do Centro de Ensino Superior de Valença, para realização de exame coproparasitológico. Foram submetidas às técnicas de centrífugo-flutuação simples e sedimentação simples, as quais revelaram positividade para Ancylostoma spp. (79,1\%), Trichuris vulpis (20,8\%), Toxocara canis (4,1\%), Dipylidium caninum $(4,1 \%)$, Capillaria spp. $(4,1 \%)$ e Heterakis spp. (4,1\%). Os resultados deste estudo demonstraram que os cães representam uma importante fonte de contaminação de praças e vias públicas por parasitos gastrintestinais que afetam outros animais e o homem, necessitando de maior atenção de autoridades públicas em relação à saúde destes animais, visando a diminuição do risco de infecção para o homem e aos próprios animais, através da implantação de medidas educativas para conscientização da população quanto a este problema de saúde pública.
\end{abstract}

Palavras chave: contaminação ambiental, endoparasitas, exame coproparasitológico

\section{Occurrence of gastrointestinal parasites in feces of dogs collected on public roads in the city of Valença-RJ}

ABSTRACT. Considering the importance of infection by gastrointestinal parasites of dogs, both in the veterinary and public health aspects, studies on the occurrence of these endoparasites present in dog feces on public roads are necessary to establish control and prophylaxis measures. Thus, the present study aimed to evaluate the occurrence of gastrointestinal parasites present in feces of dogs collected on public roads in the city of Valença -RJ from August to October 2017. Sixty-three samples of fresh dog feces were collected and they were sent to the Laboratory of Parasitology and Parasitic Diseases of Centro de Ensino Superior de Valença, for a coproparasitological examination. They were submitted to the simple centrifugation-flotation and simple sedimentation techniques, which revealed positivity for Ancylostoma spp. (79.1\%), Trichuris vulpis (20.8\%), Toxocara canis (4.1\%), Dipylidium caninum (4.1\%), Capillaria spp. (4.1\%) and Heterakis spp. (4.1\%). The results of this study demonstrated that dogs represent an important source of contamination of squares and public roads by gastrointestinal parasites that affect other 
animals and man. Thus requiring greater attention of public authorities regarding the health of these animals, aiming to reduce the risk of infection to humans and to the animals themselves, through the implementation of educational measures to raise public awareness about this public health problem.

Keywords: coproparasitological examination, endoparasites, environmental contamination

\section{Parásitos gastrointestinales en heces de perros recogidas en vías públicas del municipio de Valença - RJ}

RESUMEN. Considerando la importancia de la infección por parásitos gastrointestinales de perros, tanto en el aspecto de la clínica veterinaria como de la salud pública, estudios sobre la incidencia de estos endoparásitos, presentes en heces de perros en vías públicas, son necesarios para establecer medidas de control y profilaxis. De esta forma, el presente estudio ha tenido como objetivo evaluar la incidencia de parásitos gastrointestinales presentes en heces de perros recogidas en vías públicas del municipio de Valença -RJ en el período de agosto a octubre de 2017. Un total de 63 muestras de heces frescas de perro fueron recogidas y encaminadas para Laboratorio de Parasitología y Enfermedades Parasitarias del Centro de Enseñanza Superior de Valença, para el análisis de coproparasitológico. Se sometieron a las técnicas de centrífugo-flotación simple y sedimentación simple, las cuales revelaron positividad para Ancylostoma spp. (79,1\%), Trichuris vulpis (20,8\%), Toxocara canis $(4,1 \%)$, Dipylidium caninum $(4,1 \%)$, Capillaria spp. $(4,1 \%)$ y Heterakis spp. (4,1\%). Los resultados de este estudio demuestran que los perros representan una importante fuente de contaminación de plazas y vías públicas por parásitos gastrointestinales que acomete otros animales y el hombre, necesitando una mayor atención de las autoridades públicas en relación a la salud de estos animales, con el fin de disminuir el riesgo de infección para el hombre y los propios animales, a través de la implantación de medidas educativas para concientización de la población en cuanto a este problema de salud pública.

Palabras clave: análisis de materia fecal, contaminación ambiental, parásitos gastrointestinales

\section{Introdução}

Os cães convivem com o ser humano há pelo menos dez mil anos (Lima \& Luna, 2012). Sua população, atualmente, é estimada em mais de 500 milhões de animais, estando presentes em todos os continentes devido a sua associação com o homem (Macpherson et al., 2005). Esta relação de convívio pode estabelecer benefícios psicológicos, fisiológicos e sociais aos seres humanos, porém em contrapartida, quando a criação desses animais é inadequada, causam alteração dos padrões de bem-estar animal, possibilidade de transmissão de doenças, ocorrências de acidentes, agressões e contaminação ambiental (Armstrong \& Botzler, 2008).

$\mathrm{O}$ rápido amadurecimento sexual, a concepção de numerosas proles, a falta de medidas políticas eficazes de controle populacional de cães e a falta de orientação sobre a guarda responsável para cidadãos que desejam conviver com esses animais, o aumento excessivo da população humana e a falta de condições de educação e higiene propiciam inúmeras condições adversas, que podem gerar o crescente número de animais abandonados, aumentando os riscos que esses animais podem apresentar para a sociedade em termos de saúde pública e desequilíbrio ambiental (Broom, 1991; Beaver, 2001; Thrusfield, 2004).

Por todo o mundo, as parasitoses intestinais são bastante frequentes entre os animais, tanto em áreas rurais quanto urbanas (El-Shehabi et al., 1999; Choo et al., 2000). Os animais acometidos podem apresentar sinais clínicos variáveis de acordo com a espécie e a carga parasitária (Brener et al., 2005). É mais comum a ocorrência de sintomatologia gastrintestinal, como diarreia, anorexia, perda de peso, anemia e desidratação. Deficiências do crescimento e morte podem ocorrer em casos mais severos (Barutzki \& Schaper, 2003).

Diferentes parasitas gastrintestinais podem ser encontrados em fezes de cães, sendo os mais comuns Ancylostoma spp., Toxocara canis e 
Trichuris vulpis (Farias et al., 1995; Scaini et al., 2003; Blazius et al, 2005; Capuano \& Rocha, 2006; Oliveira et al., 2009; Leite, 2013; Mergener et al., 2013; Alves et al., 2014). Podem ser encontrados também com menor frequência os Strongyloides spp. (Scaini et al., 2003), Dipylidium caninum (Blazius et al., 2005; Oliveira et al., 2009; Mergener et al., 2013), Echinococcus granulosus, Giardia intestinalis (Mergener et al., 2013) e Cystoisospora spp. (Blazius et al., 2005; Capuano \& Rocha, 2006).

Sem a execução de programas de sanidade animal que objetivem o uso de antiparasitários como rotina, esses animais ficam permanentemente expostos à ação de parasitos, constituindo uma importante fonte de risco à saúde humana (Leite, 2013). Dessa forma, o fácil acesso destes animais, nessas condições, às ruas, praças, parques e outros logradouros públicos, sejam domiciliados, peridomiciliados, abandonados ou errantes, contribui de maneira fundamental no aumento da contaminação desses locais (Scaini et al., 2003), uma vez que as fezes desses animais contaminam o solo com vários tipos e formas parasitárias potencialmente causadoras de zoonoses (Vargas et al., 2013). Algumas dessas zoonoses são capazes de gerar efeitos variáveis de leves a graves para a saúde humana, embora necessitem de abordagem terapêutica e até de internações hospitalares, principalmente os indivíduos mais vulneráveis, como crianças, idosos e pessoas subnutridas (Chen \& Mucci, 2012).

Dentre as parasitoses de caráter zoonótico, destacam-se as formas larvares de Ancylostoma caninum e A. brasiliensis que penetram na pele do homem e vagueiam no tecido subcutâneo provocando uma erupção linear e tortuosa da pele, geralmente muito pruriginosa denominada Larva Migrans Cutânea - LMC (Nunes et al., 2000); a Larva Migrans Visceral - LMV, que o homem adquire através da ingestão de ovos contendo a larva de segundo estágio do Toxocara spp. Esta penetra na parede do intestino, após eclodir do ovo, e inicia uma migração para o fígado, pulmão e outros órgãos, causando sinais e sintomas variáveis de acordo com a localização da larva, sendo os mais comuns: febre, problemas respiratórios e hepatomegalia (Anaruma Filho et al., 2003); Echinococcus granulosus, cujas formas imaturas causam o cisto hidático; Dipylidium caninum e Strongyloides stercoralis, que são capazes de provocar infecção intestinal no homem (Labruna et al., 2006).

Dentre os protozoários, destacam-se Giardia intestinalis e Cryptosporidium spp., que também podem causar infecção intestinal no homem (Beneson, 1977; Long, 1990).

A contaminação de locais como parques, ruas, praças e outros logradouros públicos por ovos de helmintos parasitos de animais de estimação é um grave problema sanitário de ordem mundial, que se agrava devido a utilização desses locais, por crianças, como áreas de lazer (Leite, 2013).

O elevado índice de ovos de endoparasitas encontrados, em fezes coletadas em vias públicas, em trabalhos realizados por Capuano \& Rocha (2006), Alves et al. (2014) e Júnior et al. (2015) evidenciam o risco potencial de transmissão de zoonoses às pessoas que estão expostas através do contato direto com animais de estimação.

Como fator agravante, a debilidade física verificada em animais parasitados nem sempre é evidente, o que torna a realização do diagnóstico coproparasitológico para o conhecimento da frequência desses enteroparasitos de grande importância, tanto pelo caráter zoonótico quanto pelos danos diretos que estes causam em seus hospedeiros. Atentando a necessidade de adoção de medidas profiláticas para seu controle, a fim de se evitar a possibilidade de transmissão para crianças, idosos e pessoas imunocomprometidas, visando uma melhor qualidade de vida para humanos e cães. (Alves et al., 2005).

O diagnóstico coproparasitológico é o recurso laboratorial mais utilizado para detecção de infecções parasitárias gastrintestinais (SouzaDantas et al., 2007), para este deve-se escolher a técnica diagnóstica de acordo com seu grau de confiabilidade e sensibilidade, além de necessitar de recursos menos onerosos (Carli, 1994), e é comum utilizar-se mais de um método de diagnóstico para detecção de formas parasitárias de helmintos e protozoários, principalmente nos casos em que há baixa carga parasitária (Mendes et al., 2005), associando uma técnica de sedimentação com uma de flutuação, o que aumenta a chance de ter um diagnóstico mais fidedigno do paciente.

As técnicas de flutuação têm como princípio a flutuação de ovos de nematoides e oocistos de protozoários em soluções com peso específico superior a dos ovos dos parasitas como a de centrifugo-flutuação simples (Figueiredo et al., 
1984). Por outro lado, as técnicas de sedimentação são indicadas para a recuperação de ovos pesados que não flutuem em soluções saturadas, como a técnica de sedimentação simples (Hoffman et al., 1934).

Considerando a importância da infecção por parasitas gastrintestinais em cães, tanto no aspecto da clínica veterinária como da saúde pública, estudos de prevalência são necessários para se estabelecer medidas de controle e profilaxia, como medidas efetivas de saúde pública (Vasconcellos et al., 2006).

O objetivo deste trabalho foi avaliar a ocorrência de parasitas gastrintestinais presentes em fezes coletadas em praças e vias públicas no município de Valença -RJ no período de agosto a setembro de 2017.

\section{Material e métodos}

\section{Coleta de Amostras}

No período de agosto a outubro de 2017 , foram coletadas 63 amostras fecais de cães em praças e vias públicas localizadas na zona central do município de Valença, RJ; o critério de escolha destes locais baseou-se no fato de apresentarem maior circulação de pessoas. As amostras foram coletadas com luvas, e auxílio de espátulas, sendo transferidas para coletores universais estéreis previamente identificados e encaminhadas, sob refrigeração, ao Laboratório de Parasitologia e Doenças Parasitárias do Centro de Ensino Superior de Valença, localizado no município de Valença, RJ.

Foram selecionadas apenas as fezes frescas, ou seja, aquelas recém-eliminadas ou que não apresentavam ressecamento e/ou crescimento de fungos decompositores. $\mathrm{O}$ reconhecimento das amostras como oriundas de cães levou em consideração características qualitativas, dentre as quais aparência, tamanho e odor.

\section{Processamento das Amostras}

Para a realização dos exames coproparasitológicos, foram empregados os métodos de centrífugo-flutuação simples (Figueiredo et al., 1984), e sedimentação simples (Hoffman et al., 1934).

Para cada método realizado, foi confeccionada uma lâmina de cada amostra, sobre as quais, no método de sedimentação simples, foram adicionadas gotas de lugol e analisadas em microscópio óptico nos aumentos de 10x e 40x.

Caso houvesse amostras consideradas positivas para coccídios, estas seriam armazenadas em solução aquosa de dicromato de potássio a 2,5\% (K2Cr2O7) na proporção de uma parte de fezes para cinco de solução (1:5), sendo mantidas em temperatura ambiente para avaliação semanal, a fim de se esperar o período de esporulação dos mesmos para posterior identificação.

\section{Resultados}

Das 63 amostras, $24(38,1 \%)$ apresentaram positividade para ovos de parasitas gastrintestinais, sendo 79,1\% (19/24) com contaminação simples (apenas um gênero/espécie por animal/amostra) e 20,8\% (5/24) contaminação mista (ovos diferentes em uma mesma amostra). Foram verificados ovos de Ancylostoma spp., Trichuris vulpis, Toxocara canis, Dipylidium caninum, Heterakis gallinarum e Capillaria spp. (Figura 1).

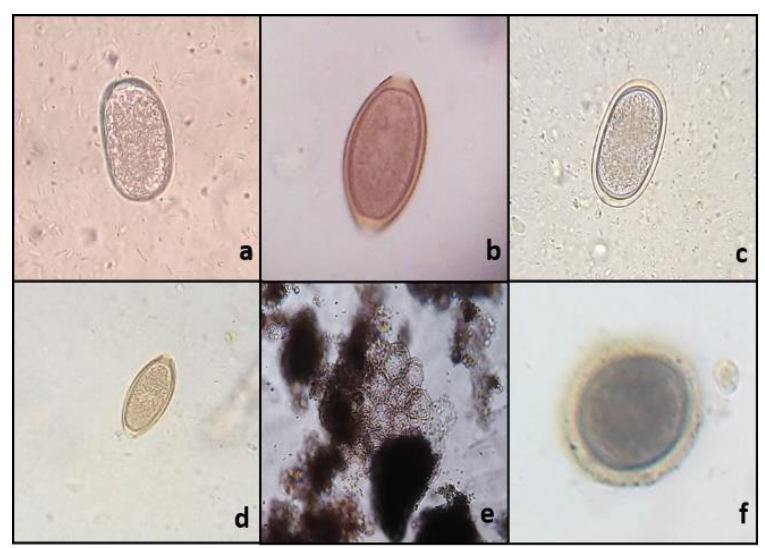

Figura 1. Ovos de helmintos identificados nas amostras fecais de cães coletadas em vias públicas: Ancylostoma spp. (a), Trichuris vulpis (b), Heterakis spp. (c), Capillaria spp. (d), Dipylidium caninum (e) e Toxocara canis (f). (Objetiva 40x).

A Figura 2 revela a quantificação de parasitas, por gênero ou espécie, verificados nas amostras positivas. O gênero de maior ocorrência foi Ancylostoma spp. verificado em $79,1 \%$ das amostras positivas, seguido por Trichuris vulpis detectado em 20,8\% das amostras, sendo a associação destes dois a principal encontrada neste estudo, em $80 \%$ das amostras contendo infecção mista, como demonstrado na Figura 3.

Neste estudo, o método de Centrífugoflutuação simples se mostrou mais sensível para identificação de ovos de parasitas gastrintestinais 
em fezes de cães que o método de Sedimentação simples, onde, com a utilização do primeiro foi possível observar a presença de ovos em 23 das 63 amostras e no segundo método apenas 4 amostras foram consideradas positivas.

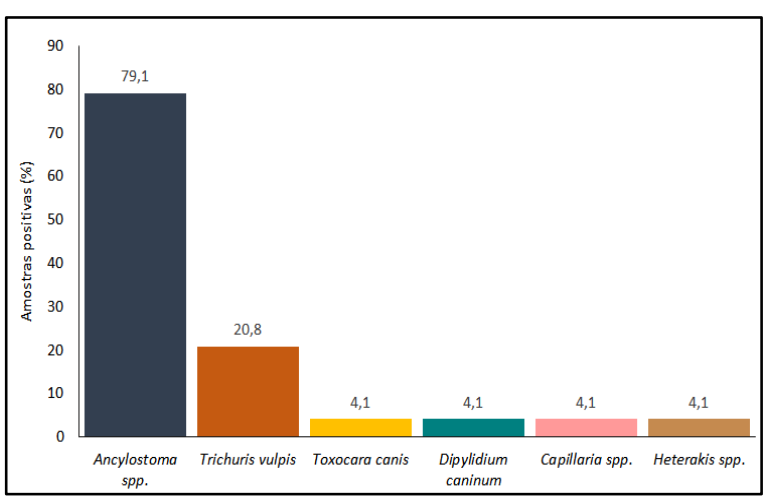

Figura 2. Quantificação de parasitas, por gênero ou espécie, nas amostras positivas (Objetiva 40x).

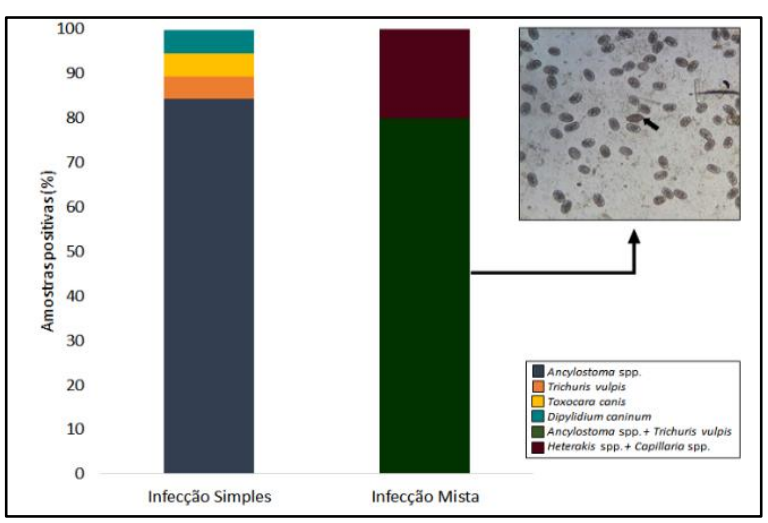

Figura 3. Percentual de parasitas gastrintestinais identificados em amostras positivas contendo infecções simples e mista. Foto de ovos de Trichuris vulpis (seta) e Ancylostoma ssp., identificados pela técnica de centrífugoflutuação simples (Objetiva 40x).

\section{Discussão}

O percentual de amostras positivas para ovos de parasitas gastrintestinais encontrado neste estudo $(38,1 \%)$ foi inferior aos $52,77 \%$ obtidos por Leite (2013), 56,8\% de Capuano \& Rocha (2006) e 51,7\% em Junior et al. (2015). Onde ambos utilizaram coleta de fezes em vias públicas, mas com técnicas coproparasitológicas diferentes.

O parasita que apresentou maior frequência foi o gênero Ancylostoma $(79,1 \%)$, corroborando a literatura que o aponta como frequente na endofauna parasitária de canídeos silvestres e domésticos (Blazius et al., 2005). Comparando com trabalhos semelhantes em que as amostras também foram coletadas em vias públicas, mas com a utilização de técnicas coproparasitológicas diferentes, pode-se concluir que o Ancylostoma spp. é o parasita mais frequentemente encontrado em fezes de cães de diferentes regiões do país como no estudo realizado no Balneário Cassino RS por Scaini et al. (2003), onde encontraram a prevalência de $71,3 \%$ amostras positivas para este gênero; em Pindamonhangaba-SP, realizado por Alves et al. (2014) com 23,4\%; em Natal-RN por Júnior et al. (2015) com 45\%; em Ribeirão PretoSP por Capuano \& Rocha (2006) com 41,7\%; e em Guarapuava-PR por Leite (2013) com 26,05\%.

A alta prevalência de Ancylostoma spp. pode ser justificada por dois principais motivos, devido à grande tolerância dos estágios de vida livre às diferentes condições ambientais, explicando a ampla distribuição geográfica destes parasitas; e devido a capacidade destes de infectarem cães de todas as faixas etárias, já que, de acordo com Boag et al. (2003), os mesmos não desenvolvem imunidade contra antígenos de Ancylostoma caninum. Neste caso, como os animais permanecem susceptíveis às infecções ao longo de toda a vida, tanto as larvas presentes no ambiente como as retidas nos tecidos podem alcançar periodicamente o intestino recolonizando-o com vermes adultos. Com isso, teoricamente, toda a população de cães contribui de forma contínua para a contaminação ambiental.

A baixa frequência observada de Trichuris vulpis, quando comparada à frequência de Ancylostoma spp., corrobora os resultados encontrados em outros estudos realizados por Blazius et al. (2005), Almeida et al. (2007) e Chen \& Mucci (2012). Este resultado pode estar relacionado com o período pré-patente do parasito em questão, que é de seis a doze semanas, considerado longo quando comparado aos parasitas do gênero Ancylostoma, que é de uma a duas semanas, e com a ovipostura, que no Trichuris vulpis é intermitente, implicando possíveis resultados falso-negativos quando da avaliação de apenas uma amostra (Elsemore et al., 2014).

A frequência de amostras positivas para o gênero Toxocara foi inferior ao esperado, contrastando com resultados de alguns trabalhos que mostraram positividade superior para ovos deste parasito, como demonstrado por Scaini et al. (2003), Capuano \& Rocha (2006) e Alves et al. (2014), que encontraram a prevalência de 9,3\%, $24,2 \%$ e $8,1 \%$, respectivamente. A principal hipótese que justificaria este achado seria a de que as fezes coletadas seriam provenientes de cães adultos, que já apresentam resposta imunitária 
efetiva contra estes parasitas, diferente dos animais jovens de até 1 ano de idade como verificado por Fischer (2003).

O encontro de ovos de Ancylostoma spp. e Toxocara canis, tem uma importância epidemiológica significante pois representam risco para a saúde pública, devido a possibilidade da ocorrência da Larva Migrans Cutânea (LMC) e da Larva Migrans Visceral (LMV).

Parasitas da espécie Dipylidium caninum, são comumente encontrados parasitando o intestino delgado de cães, representando importância para a saúde dos cães e do homem, pois constitui uma zoonose. Como o potencial de transmissão é relacionado a densidade populacional de pulgas, que são hospedeiras intermediárias do parasita, as prevalências são variáveis de acordo com a infestação por esses ectoparasitas. A baixa prevalência de Dipylidium caninum $(4,3 \%)$ encontrada neste estudo já era esperada, pois este parasita elimina proglotes ao invés de ovos, dificultando seu diagnóstico por exames coproparasitários. Um aspecto que se destaca quando se examina a literatura relativa à prevalência de $D$. caninum é a discrepância das prevalências registradas: extremamente elevadas quando o diagnóstico é feito pelo encontro dos vermes à necropsia (Eguía-Aguilar et al., 2005) e muito baixas quando detectada por exames coproparasitológicos (Oliveira-Sequeira et al., 2002; Ramírez-Barrios et al., 2004). A constatação de discrepâncias na prevalência desse parasita, em uma mesma região geográfica, devido à metodologia diagnóstica, tem incentivado a pesquisa de métodos sorológicos para diagnóstico dessa parasitose (Shin \& Liao, 2002).

Um achado incomum deste estudo foi a presença de ovos de Heterakis spp., que é um parasito não habitual de cães. Como este parasita infecta comumente aves silvestres e domésticas (Radfar et al., 2012; Wongrak et al., 2014) acredita-se que a amostra positiva para este parasita era proveniente de um cão que contactuava ou ingeriu vísceras cruas destas aves, sendo esta a possível contaminação canina que explica o achado parasitário.

Os métodos de centrífugo-flutuação simples (CFS) e sedimentação simples foram escolhidos pois possuem princípios físicos diferentes, sendo complementares quanto a sensibilidade dos resultados obtidos, quando usados conjuntamente, o que teoricamente não seria observado em situações de uso isolado, garantindo o diagnóstico de ovos leves e pesados, oocistos e cistos nas fezes, quando contaminadas.

No presente trabalho ao se comparar a eficácia dos métodos de Centrífugo-flutuação simples e Sedimentação Simples para o diagnóstico de enteroparasitos, observou-se que o primeiro método se mostrou mais sensível que o segundo, tanto para a pesquisa de ovos pesados (Trichuris sp. e Toxocara sp.) quanto para a pesquisa de ovos leves (Ancylostoma spp.). No entanto, o ovo de Dipylidium caninum foi observado quando se utilizou o método de sedimentação. A associação das duas técnicas não deve ser dispensada para o diagnóstico de helmintos gastrintestinais em cães.

\section{Conclusão}

Os resultados deste estudo demonstraram que os cães representam uma importante fonte de contaminação de praças e vias públicas por parasitos gastrintestinais que afetam outros animais e o homem, necessitando de maior atenção de autoridades públicas em relação à saúde destes animais, visando a diminuição do risco de infecção para o homem e aos próprios animais, através da implantação de medidas educativas para conscientização da população quanto a este problema de saúde pública.

A contaminação ambiental por ovos de parasitas gastrintestinais de animais de estimação em locais como parques recreativos, praças e vias públicas é um grave problema sanitário de ordem mundial, que é agravado pela presença de crianças utilizando esses locais para lazer.

$\mathrm{O}$ alto índice de amostras fecais positivas para parasitas gastrintestinais avaliadas no presente estudo evidencia o potencial risco de transmissão de zoonoses a que estão expostas pessoas que tem contato direto com animais de estimação. Um fator agravante é que a debilidade física nem sempre é verificada de forma evidente em animais com infectados.

A avaliação coproparasitológica de rotina realizada para detectar parasitas gastrintestinais em animais de estimação, associada ao uso racional de anti-helmínticos de amplo espectro e princípios ativos de alta qualidade contribuem no combate às parasitoses de cães e gatos. Por outro lado, a alta resistência dos ovos no ambiente e a dificuldade de desinfecção justificam a 
necessidade da implementação de medidas para a efetiva descontaminação do solo.

Elucida-se aos proprietários de cães, que os levam para passear, que recolham as fezes de seus animais com sacolas higiênicas após defecação em vias públicas.

\section{Referências bibliográficas}

Almeida, A. B. P. F., Sousa, V. R. F., Dalcin, L. \& Justino, C. H. S. 2007. Contaminação por fezes caninas das praças públicas de Cuiabá, Mato Grosso. Brazilian Journal of Veterinary Research and Animal Science, 44, 132-136.

Alves, A. P. S., Coêlho, F. A. S. \& Coêlho, M. D. G. 2014. Frequência de enteroparasitos em fezes de cães coletadas em praças públicas do município de Pindamonhangaba-SP, Brasil. Revista de Patologia Tropical, 43, 341-350.

Alves, O. F., Gomes, A. G. \& Silva, A. C. 2005. Ocorrência de Enteroparasitos em Cães do Município de Goiânia, Goiás: Comparação de Técnicas de Diagnóstico. Ciência Animal Brasileira, 6, 127-133.

Anaruma Filho, F., Chieffi, P. P., Correa, R. R. S., Camargo, E. D. Silveira, E. P. R. \& Aranha, J. J. B. 2003. Human toxocariasis: incidence among residents in the outskirts of Campinas, state of São Paulo, Brazil. Revista do Instituto de Medicina Tropical, 45, 293-294.

Armstrong, S. J. \& Botzler, R. G. 2008. The animal ethics reader. Routledge, London, UK.

Barutzki, D. \& Schaper, R. 2003. Endoparasites in dogs and cats in Germany 1999-2002. Parasitology Research, 90, 148-150.

Beaver, B. V. 2001. Comportamento canino: um guia para Veterinários. Rocca, São Paulo, BR.

Blazius, R. D., Emerick, S., Prophiro, J. S., Romão, P. R. T. \& Silva, O. S. 2005. Ocorrência de protozoários e helmintos em amostras de fezes de cães errantes da Cidade de Itapema, Santa Catarina. Revista da Sociedade Brasileira de Medicina Tropical, 38,73-74.

Boag, P. R., Parsons, J. C., Presidente, P. J. Spithill, T. W. \& Sexton, J. L. 2003. Characterisation of humoral imune responses in dogs vaccinated with irradiated Ancylostoma caninum. Veterinary Immunology and Immunopathology, 92, 87-94.

Brener, B., Lisboa, L., Mattos, D. P. B. G., Arashiro, E. K. N., Millar, P. R., Sudré, A. P. \& Duque, V. 2005. Frequência de enteroparasitas em amostras fecais de cães e gatos dos municípios do Rio de Janeiro e Niterói. Revista Brasileira de Ciência Veterinária, 12, 102-105.

Broom, D. M. 1991. Animal Welfare: concepts and measurement. Journal of Animal Science, 69, 4167- 4175 .

Capuano, D. M. \& Rocha, G. M. 2006. Ocorrência de parasitas com potencial zoonótico em fezes de cães coletadas em áreas públicas do município de Ribeirão Preto, SP, Brasil. Revista Brasileira de Epidemiologia, 9, 81-6.

Carli, G. A. 1994. Diagnóstico laboratorial das parasitoses humanas, métodos e técnicas. Medsi, Rio de Janeiro, BR.

Chen, A. A. \& Mucci, J. L. N. 2012. Frequência de contaminação por helmintos em área de recreação infantil creches no município de Várzea Paulista, São Paulo, Brasil. Revista de Patologia Tropical, 41, 195-202.

Choo, J., Pang, E. \& Prociv, P. 2000. Hookworms in dogs of Kuching, Sarawak (north Borneo). Transactions of the Royal Society of Tropical Medicine and Hygiene, 94, 21-22.

Eguía-Aguilar, P., Cruz-Reyes, A. \& MartínezMaya, J. J. 2005. Ecological analysis and description of the intestinal helminthes present in dogs in Mexico City. Veterinary Parasitology, 127, 139-146.

Elsemore, D. A, Geng, J., Flynn, L., Cruthers, L., Lucio-Forster, A. \& Bowman, D. D. 2014. Enzyme-linked immunosorbent assay for coproantigen detection of Trichuris vulpis dogs. Journal of Veterinary Diagnostic Investigation, 26, 404-411.

El-Shehabi, F. S., Abdel-Hafez, S. K. \& Kamhawi, S. A. 1999. Prevalence of intestinal helminthes of dogs and foxes from Jordan. Parasitology Research, 85, 928-934.

Farias, N. A., Christovão, M. I. \& Stobbe, N. S. 1995. Frequência de parasitos intestinais em cães (Canis familiaris) e gatos (Felis catus domestica) em Araçatuba- São Paulo. Revista Brasileira de Parasitologia Veterinária, 4, 5760.

Figueiredo, P. C., Serra-Freire, N. M. \& Grisi, L. 1984. Eimerias de bovinos leiteiros no Estado do Rio de Janeiro: técnica de diagnóstico e espécies identificadas. Atas Sociedade de Biologia do Rio de Janeiro, 24, 22-26.

Fischer, C. D. B. 2003. Prevalência de helmintos em Canis familiaris (Linnaeus, 1758) no 
Hospital de Clinicas Veterinárias do Rio Grande do Sul através de diagnóstico postmortem. Acta Scientiae Veterinariae, 31, 6364.

Hoffman, W. A., Pons, J. A. \& Janer, J. L. 1934. Sedimentation concentration method in schistosomiasis mansoni. Puerto Rico Journal of Public Health and Tropical Medicine, 9, 283-298.

Júnior, A. L. F. A., Araújo, K. B. S. \& Medeiros, V. S. 2015. Ocorrência de Parasitas com Potencial Zoonótico em Fezes de Cães Coletadas em Vias Públicas da Cidade De Natal. Revista Humano Ser - UNIFACEX, 1, 52-59.

Labruna, M. B., Pena, H. F. J., Souza, S. L. P., Pinter, A., Silva, J. C. R. Ragozo, M. M. A., ... \& Gennari, S.M. 2006. Prevalência de Endoparasitas em Cães da Área Urbana do Município de Monte Negro, Rondônia. Arquivos do Instituto Biológico, 73, 183-193.

Leite, L. C. 2013. Ocorrência de ovos de endoparasitas em amostras de fezes de cães (Canis familiaris, Linnaeus, 1758) coletadas em vias públicas da cidade de Guarapuava Paraná - Brasil. Ambiência - Revista do Setor de Ciências Agrárias e Ambientais, 9, 619626.

Lima, A. F. M. \& Luna, S. P. L. 2012. Algumas causas e consequências da superpopulação canina e felina: acaso ou descaso? Revista de Educação Continuada em Medicina Veterinária e Zootecnia do CRMV-SP, 10, 3238.

Long, P. L. 1990. Coccidiosis of man and animals. CRC Press, Boca Raton, US.

Macpherson, C. N. L. 2005. Human behavior and the epidemiology of parasitic zoonoses. International Journal for Parasitology, 35, 319-1331.

Mendes, C. R., Teixeira, A. T. L. S., Pereira, R. A. T. \& Dias, L. C.S. 2005. Estudo comparativo entre os métodos de Kato-Katz e coprotest. Revista da Sociedade Brasileira de Medicina Tropical, 38, 178-180.

Mergener, D., Caricimo, A., Langer D., Klein, D., Lima, G., Verona, J., .. \& Muller, G. A. 2013. Enteroparasitos de Cães Errantes da Zona Urbana dos Municípios de Água Doce, Irani, Joaçaba, Ponte Serrada e Treze Tílias, Santa Catarina, Brasil. Unoesc \& Ciência - ACBS, 4,127-134.
Nunes, C. M., Pena, C. P., Negrelli, G. B., Anjo, C. G. S., Nakano, M. M. \& Stobbe, N. S. 2000. Ocorrência de larva migrans na areia de áreas de lazer das escolas municipais de ensino infantil, Araçatuba, SP, Brasil. Revista de Saúde Pública, 34, 656-658.

Oliveira, V. S. F., Melo, D. P. G., Fernandes, P. R., Schulze, C. M. B., Guimarães, M. S. \& Silva, A. C. 2009. Ocorrência de helmintos gastrintestinais em cães errantes na cidade de Goiânia - Goiás. Revista de Patologia Tropical, 38, 279-283.

Oliveira-Sequeira, T. C. G., Amarante, A. F., Ferrari, T. B. \& Nunes, L. C. 2002. Prevalence of intestinal parasites in dogs from São Paulo State, Brazil. Veterinary Parasitology, 103, 1927.

Radfar, M. H., Khedri, J., Adinehbeigi, K. \& Rahmani, K. 2012. Columba livia domestica and free-range backyard chickens of Sistan region, east of Iran. Journal of Parasitic Diseases, 36, 220-225.

Ramírez-Barrios, R. A., Barboza-Mena, G., Munoz, J., Angulocubillan, F., Hernandez, E., Gonzalez, F. \& Escalona, F. 2004. Prevalence of intestinal parasites in dogs under veterinary care in Maracaibo, Venezuela. Veterinary Parasitology, 121, 11-20.

Scaini, C. J., Toledo, R. C., Lovatel, R., Dionello, M. A.; Gatti, F. A.; Susin, L. \& Signorini, V. R. M. 2003. Contaminação ambiental por ovos e larvas de helmintos em fezes de cães na área central do Balneário Cassino, Rio Grande do Sul. Revista da Sociedade Brasileira de Medicina Tropical, 36, 617-619.

Shin, J. W. \& Liao, W. T. 2002. Humoral immune response to Dipylidium caninum infection of stray dogs in Taiwan. Veterinary Parasitology, 104, 351-356.

Souza-Dantas, L. M., Bastos, O. P. M., Brener, B., Salomão, M., Guerrero, J. \& Labarthe, N. V. 2007. Técnica de centrífugo-flutuação com sulfato de zinco no diagnóstico de helmintos gastrintestinais de gatos domésticos. Ciência Rural, 37, 904-906.

Thrusfield, M. 2004. Epidemiologia veterinária. Roca, São Paulo, BR.

Vargas, M. M., Bastiani, M. \& Ferreira, J. R. D. 2013. Frequência de estruturas parasitárias em praças e parques públicos da cidade de Porto Alegre-RS. Revista de Patologia Tropical, 42, 434-442. 
Vasconcellos, M. C., Barros, J. S. L. \& Oliveira, C. S. 2006. Parasitas gastrointestinais em cães institucionalizados no Rio de Janeiro, RJ. Revista de Saúde Pública, 40, 321-323.

Wongrak, K., Das, G., Moors, E., Sohnrey, B. \& Gauly, M. 2014. Establishment of gastrointestinal helminth infections in free-range chickens: a longitudinal on farm study. Berl Munch Tierarztl Wochenschr, 127, 314-321.
Recebido: 7 Jul., 2018.

Aprovado: 14 Ago., 2018

Publicado: 24 Ago., 2018

Licenciamento: Este artigo é publicado na modalidade Acesso Aberto sob a licença Creative Commons Atribuição 4.0 (CC-BY 4.0), a qual permite uso irrestrito, distribuição, reprodução em qualquer meio, desde que o autor e a fonte sejam devidamente creditados. 